\title{
Modelling adsorption using an augmented two-dimensional statistical associating fluid theory: 2D SAFT-VR Mie
}

\author{
Gerardo Campos-Villalobos, ${ }^{1,}{ }^{*}$ Srikanth Ravipati, ${ }^{2,} \dagger$ Andrew Haslam, ${ }^{2,} \ddagger$ \\ George Jackson, ${ }^{2, \S}$ Jonatan Suaste, ${ }^{3,}$ ฯ and Alejandro Gil-Villegas ${ }^{3, * *}$ \\ 1 Department of Chemical Engineering and Analytical Science, \\ University of Manchester, Sackville Street, Manchester M13 9PL, U.K. \\ ${ }^{2}$ Department of Chemical Engineering, Imperial College London, London SW7 2AZ, U.K. \\ ${ }^{3}$ División de Ciencias e Ingenierías, Campus León, \\ Universidad de Guanajuato, Loma del Bosque 103, \\ Lomas del Campestre, 37150 León, Guanajuato, México
}

\begin{abstract}
We present an extension of the SAFT-VR Mie approach to model adsorption of molecular fluids based on a two-dimensional (2D) approximation to describe the adsorbed fluid. Analytical results are provided for the first- and second-order perturbation terms of the $2 \mathrm{D}$ system. The adsorption model is based on the assumption that the particle pair interactions in the adsorbed and bulk phases are described with the same Mie potential parameters $\lambda_{\mathrm{a}}$ and $\lambda_{\mathrm{r}}$, in contrast with the square-well version of the 2D-SAFT-VR approach in which it is considered necessary to modify the attractive ranges of the SW interactions. This important difference between theories reduces the number of molecular parameters to be determined. In order to demonstrate the performance of the 2D SAFTVR-Mie approach, we present results for the the modelling of $\mathrm{CO}_{2}$ and $\mathrm{CH}_{4}$ adsorbed onto dry coal.

PACS numbers:
\end{abstract}

\section{INTRODUCTION}

Adsorption of fluids onto solid surfaces is a common process in nature that can be rationalized in terms of the intermolecular interactions between fluid molecules and the molecules comprising the solid surface. These interactions, as well as the geometric constraining effect of the adsorbent surface, induce an inhomogeneous ordering of molecules of the adsorbed fluid.

Adsorption plays an important role in many industrial processes, including: heterogeneous catalysis, where a fluid reacts on the surface of a solid catalyst; development of nanoporous materials to separate contaminants from waste streams; hydrogen storage in cells [1]. Adsorption is particularly important in enhanced-oil-recovery (EOR), wherein there is selective adsorption of water, oil, gas and other EOR fluids into the pores present in soils and rocks. Hence, to improve the efficiency of EOR processes, a fundamental understanding of the phase behaviour of confined fluids is needed. Carbon storage takes place in the EOR process and a molecular description of the adsorption phenomena is required in order to understand and predict the thermodynamic properties of the fluid under confinement. The main purpose of the carbon-dioxide storage via adsorption is to remediate the

\footnotetext{
*Electronic address: gerardodejesus.camposvillalobos@ manchester.ac.uk

$\dagger$ Electronic address: s.ravipati@imperial.ac.uk

${ }^{\ddagger}$ Electronic address: a.haslam@imperial.ac.uk

$\S$ Electronic address: g.jackson@ic.ac.uk

IElectronic address: suastemj2010@licifug.ugto.mx

**Electronic address: gil@fisica.ugto.mx
}

greenhouse effect due to accumulation of gases in the atmosphere and, at the same time, to enhance the process of oil recovery. In 2013, $\mathrm{CO}_{2}$ accounted for about 82 percent of all U.S. greenhouse-gas emissions from human activities [2].

In the statistical models for adsorption phenomena, confinement effects are taken into account by considering a fluid formed by particles or molecules in the presence of surfaces that act as external potentials. As a consequence of this external interaction, molecules adsorb onto the surfaces, and molecular equations of state (EOS) can be provided to describe the thermodynamic properties of the fluid near the wall. Following this approach, Langmuir [3] and Brunauer, Emmett and Teller [4] developed the first theoretical predictions of adsorption isotherms for monolayers and multilayers, respectively. The BET method provided a route still used today for the determination of the surface area of the adsorbent surface $[5,6]$. The BET approach has also been extended to model more-complex sorption processes in fluids $[7,8]$. On the other hand, although molecular simulations can be applied to accurately describe the adsorption of complex fluids [9-11], the prediction of adsorption isotherms by these methods remains challenging due to the huge effort required in order to obtain a significant number of state points to construct a single curve. An alternative route fully explored by several authors over the years has been to model adsorbed fluids by two-dimensional EOS [12-17].

Based on the SAFT-VR approach for square-well (SW) fluids [18] and its formulation for 2D discrete-potential systems (2D-SAFT-VR) [19, 20], predictions of adsorption isotherms for molecular fluids and their mixtures have been previously reported [21-24], as well as for as- 
phaltenes in porous materials [25]. In this paper we present the improvement of the 2D-SAFT-VR adsorption theory by considering the case of the spherically symmetric Mie potential [26], which has been applied to a wide range of substances in fluid bulk conditions [27, 28].

The Mie pair potential is defined by

$$
u^{\mathrm{Mie}}(r)=C \varepsilon\left[\left(\frac{\sigma}{r}\right)^{\lambda_{\mathrm{r}}}-\left(\frac{\sigma}{r}\right)^{\lambda_{\mathrm{a}}}\right]
$$

where $\varepsilon$ is the depth of the potential well, $r$ is the internueclear distance between the spherical segments, $\sigma$ is the position at which the potential is zero, and $\lambda_{\mathrm{a}}$ and $\lambda_{\mathrm{r}}$ are the attractive and repulsive exponents, respectively; the pre-factor $C$ ensures that the minimum of the potential is $-\varepsilon$ regardless of the values of the exponents and is given by

$$
C=\frac{\lambda_{\mathrm{r}}}{\lambda_{\mathrm{r}}-\lambda_{\mathrm{a}}}\left(\frac{\lambda_{\mathrm{r}}}{\lambda_{\mathrm{a}}}\right)^{\tau} ; \quad \tau=\frac{\lambda_{\mathrm{a}}}{\lambda_{\mathrm{r}}-\lambda_{\mathrm{a}}} .
$$

For the case $\lambda_{\mathrm{r}}=12$ and $\lambda_{\mathrm{a}}=6$, the Mie potential takes the form of the well-known Lennard-Jones (LJ) model.

Recently, Franco et al. [29] proposed an equation of state for confined fluids using the SAFT-VR-Mie approach with a free-energy term due to confinement. Such an extension was obtained within the generalised van der Waals framework by assuming a SW potential for the fluid-solid interaction (as introduced in the 2D-SAFTVR approach [19]), which is integrated over the confining space considering semi-empirical models for the inhomogeneous fluid structure under different geometries.

The 2D-SAFT-VR Mie EOS presented here is based on an application of the Barker and Henderson (BH) [30] high-temperature perturbation expansion, up to secondorder, to a hard-disk reference system. After describing the adsorption model and theory in Sec. II, we start our theoretical treatment calculating analytical expressions for the first- and second-order perturbation terms using an analytical approximation to the pair correlation function of hard bodies in 2D space, a strategy recently adopted by Trejos et al. [31] and also discussed in [32]. The perturbation terms are validated by direct comparison with exact numerical results from Monte Carlo (MC) simulations. Finally in Sec. III, we describe the methodology for the parameterisation of fluid-fluid and fluid-solid interactions and test the theory on the description of adsorption of $\mathrm{CO}_{2}$ and $\mathrm{CH}_{4}$ on dry coal.

\section{THEORY}

We consider a model of a single-component fluid composed of $N$ spherical particles in the presence of a solid uniform wall, as depicted in Fig. 1. The interaction potential exerted by the wall on a particle is denoted as $u_{\mathrm{pw}}$, and it is assumed that this potential is a function only of $z$, given by,

$$
u_{\mathrm{pw}}\left(z ; \sigma, \lambda_{\mathrm{w}}\right)= \begin{cases}\infty & \text { if } z<0 \\ -\varepsilon_{\mathrm{w}} & \text { if } 0 \leq z \leq \lambda_{\mathrm{w}} \sigma \\ 0 & \text { if } z>\lambda_{\mathrm{w}} \sigma\end{cases}
$$

where $z$ is the perpendicular distance from the wall to the particle, $\lambda_{\mathrm{w}} \sigma$ is the length scale of the attractive potential and $\varepsilon_{\mathrm{w}}$ is the depth of the well. The system is comprised of two subsystems: the particles near to the wall, i.e., those for which $z \leq \lambda_{\mathrm{w}} \sigma$, represent the adsorbed phase, and the particles that are far from the wall, i.e., for which $z>\lambda_{\mathrm{w}} \sigma$, comprise the bulk phase. In the present approach, the bulk and adsorbed phases can be considered divided by a surface with null thickness, i.e., the interface has zero volume [33]. This approximation allows us to model the adsorption process in the same way as a two-phase system.

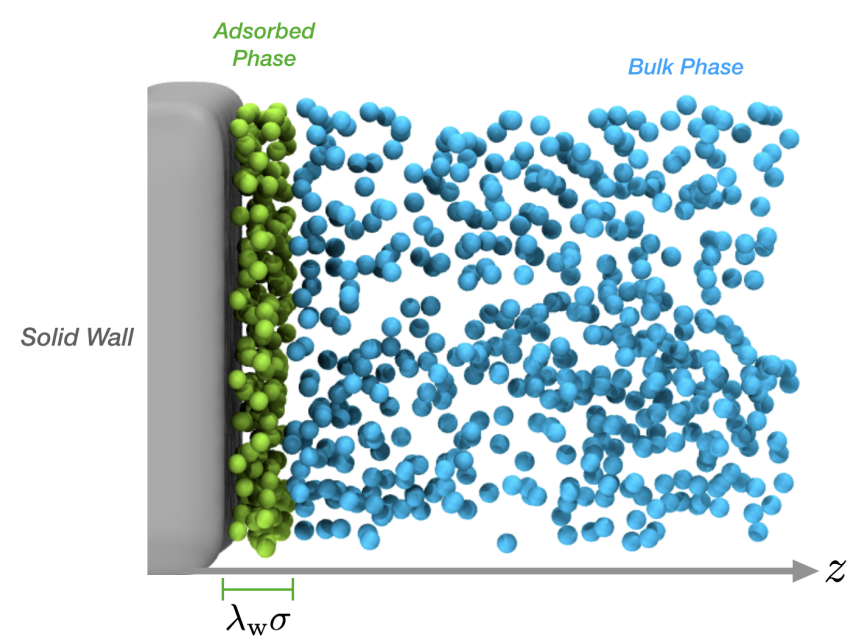

Figure 1: System of study. The system is divided into two subsystems, an adsorbed phase (green spheres) and a bulk phase (blue spheres).

Due to the presence of the wall, the adsorbed fluid exhibits different macroscopic properties in relation to the bulk fluid. For the bulk phase, the pair potential is written as $u_{\mathrm{pp}}$ and for the adsorbed phase, the molecules interact with each other via the potential denoted as $u_{\mathrm{pp}}^{\mathrm{ads}}$. For the adsorbed fluid, the pair potential interaction can be described as a decoupling of the $x, y$ coordinates from the coordinate $z$ for each adsorbed particle,

$$
\phi(x, y)=\frac{1}{\lambda_{\mathrm{w}} \sigma} \int u_{\mathrm{pp}}^{\mathrm{ads}}(x, y, z) \mathrm{d} z
$$

and the adsorbed phase can be approximated by a quasitwo dimensional system, i.e., the particles comprising the adsorbed phase interact with each other only in the $x-y$ plane. The canonical partition function of the adsorbed fluid is then given by

$$
Z_{\mathrm{ads}}=\frac{V_{\mathrm{ads}}^{N_{\mathrm{ads}}} Q_{\mathrm{ads}}}{N_{\mathrm{ads}} ! \Lambda^{3 N_{\mathrm{ads}}}}
$$


where $\Lambda$ is the de Broglie thermal wavelength, $V_{\text {ads }}$ is the volume occupied by the adsorbed molecules, $N_{\text {ads }}$ is the total number of adsorbed molecules and $Q_{\text {ads }}$ is the configurational integral of the total pair potential in the adsorbed fluid, i.e.,

$$
Q_{\mathrm{ads}}=\frac{1}{V_{\mathrm{ads}}^{N_{\mathrm{ads}}}} \int \exp \left(-\beta U_{\mathrm{tot}}\right) \mathrm{d} \mathbf{r}^{N_{\mathrm{ads}}}
$$

where $\beta=1 / k_{\mathrm{B}} T$, with $k_{\mathrm{B}}$ the Boltzmann constant and $T$ the absolute temperature. The total pair potential for the particles in the adsorbed phase is given by

$$
U_{\mathrm{tot}}=U_{\mathrm{pp}}^{\mathrm{ads}}(x, y)+U_{\mathrm{pw}}\left(z ; \sigma, \lambda_{\mathrm{w}}\right) .
$$

In this equation, $U_{\mathrm{pp}}^{\mathrm{ads}}(x, y)$ is the total potential for the adsorbed particles, i.e., $U_{\mathrm{pp}}^{\mathrm{ads}}=(1 / 2) N_{\mathrm{ads}}\left(N_{\mathrm{ads}}-1\right) \phi$, and $U_{\mathrm{pw}}$ is the total potential for the interaction of the adsorbed particles with the wall, $U_{\mathrm{pw}}=N_{\mathrm{ads}} u_{\mathrm{pw}}$. The configurational integral is then written as

$$
\begin{array}{r}
Q_{\mathrm{ads}}=\frac{1}{V_{\mathrm{ads}}^{N_{\mathrm{ads}}}} \int \exp \left(-\beta N_{\mathrm{ads}} u_{\mathrm{pw}}\left(z ; \sigma, \lambda_{\mathrm{w}}\right)\right) \mathrm{d} z^{N_{\mathrm{ads}}} \\
\quad \int \exp \left(-\beta \frac{N_{\mathrm{ads}}\left(N_{\mathrm{ads}}-1\right)}{2} \phi(x, y)\right) \mathrm{d} x^{N_{\mathrm{ads}}} \mathrm{d} y^{N_{\mathrm{ads}}} .
\end{array}
$$

Within the 2D-SAFT-VR approach [19, 20], the volume occupied by the adsorbed particles is given by the product of the adsorbed area $(s)$ and the perpendicular distance between the wall and the fluid influenced by the wall, taken as the particle-wall SW range $\lambda_{\mathrm{w}} \sigma$. As a result of the decoupling approximation,

$$
Q_{\mathrm{ads}}=Q_{1 \mathrm{D}} Q_{2 \mathrm{D}}
$$

where $Q_{1 \mathrm{D}}$ and $Q_{2 \mathrm{D}}$ are the one- and two-dimensional configurational integrals respectively, i.e.,

$$
\begin{aligned}
Q_{1 \mathrm{D}} & =\frac{1}{\left(\lambda_{\mathrm{w}} \sigma\right)^{N_{\mathrm{ads}}}} \int_{0}^{\lambda_{\mathrm{w}} \sigma} \exp \left(-\beta N_{\mathrm{ads}} u_{\mathrm{pw}}\left(z ; \sigma ; \lambda_{\mathrm{w}}\right)\right) \mathrm{d} z^{N_{\mathrm{ads}}} \\
& =\left[\frac{1}{\lambda_{\mathrm{w}} \sigma} \int_{0}^{\lambda_{\mathrm{w}} \sigma} \exp \left(-\beta u_{\mathrm{pw}}\left(z ; \sigma, \lambda_{\mathrm{w}}\right)\right) \mathrm{d} z\right]^{N_{\mathrm{ads}}}
\end{aligned}
$$

$Q_{2 \mathrm{D}}=\frac{1}{s^{N_{\mathrm{ads}}}} \int \exp \left(-\beta \frac{N_{\mathrm{ads}}\left(N_{\mathrm{ads}}-1\right)}{2} \phi(x, y)\right) \mathrm{d} x^{N_{\mathrm{ads}}} \mathrm{d} y^{N_{\mathrm{ads}}}$

The integral in Eq. (9) can be evaluated using the mean-value theorem as

$$
Q_{1 \mathrm{D}}=\exp \left(-\beta N_{\mathrm{ads}} u_{\mathrm{pw}}\left(z^{*} ; \sigma, \lambda_{\mathrm{w}}\right)\right)
$$

where $z^{*}$ is the value of the $z$ coordinate that guarantees the mean value of $Q_{1 \mathrm{D}}$. The canonical partition function for the adsorbed fluid is thus given by the expression

$$
Z_{\mathrm{ads}}=Z_{\mathrm{ads}}^{1 \mathrm{D}} Z_{\mathrm{ads}}^{2 \mathrm{D}}
$$

where

$$
Z_{\mathrm{ads}}^{1 \mathrm{D}}=\frac{\left(\lambda_{\mathrm{w}} \sigma\right)^{N_{\mathrm{ads}}}}{\Lambda^{N_{\mathrm{ads}}}} \exp \left(-\beta N_{\mathrm{ads}} u_{\mathrm{pw}}\left(z^{*} ; \sigma, \lambda_{\mathrm{w}}\right)\right)
$$

and

$$
\begin{aligned}
Z_{\mathrm{ads}}^{2 \mathrm{D}} & =\frac{s^{N_{\mathrm{ads}}}}{N_{\mathrm{ads}} ! \Lambda^{2 N_{\mathrm{ads}}}} \\
& \times \int \exp \left(-\beta \frac{N_{\mathrm{ads}}\left(N_{\mathrm{ads}}-1\right)}{2} \phi(x, y)\right) \mathrm{d} x^{N_{\text {ads }}} \mathrm{d} y^{N_{\text {ads }}} .
\end{aligned}
$$

Rearranging Eqs. (12)-(14) we obtain

$$
Z_{\mathrm{ads}}=Z_{\mathrm{ads}}^{2 \mathrm{D}}\left(\frac{\lambda_{\mathrm{w}} \sigma}{\Lambda}\right)^{N_{\mathrm{ads}}} \exp \left(-\beta N_{\mathrm{ads}} u_{\mathrm{pw}}\left(z^{*} ; \sigma, \lambda_{\mathrm{w}}\right)\right) .
$$

The Helmholtz free energy of the adsorbed fluid is then given by $A_{\mathrm{ads}}=-k_{\mathrm{B}} T \ln Z_{\mathrm{ads}}$ :

$$
\frac{A_{\mathrm{ads}}}{N_{\mathrm{ads}} k_{\mathrm{B}} T}=\frac{A_{2 \mathrm{D}}}{N_{\mathrm{ads}} k_{\mathrm{B}} T}-\ln \left(\frac{\lambda_{\mathrm{w}} \sigma}{\Lambda}\right)+\beta u_{\mathrm{pw}}\left(z^{*} ; \sigma, \lambda_{\mathrm{w}}\right)
$$

where the term $u_{\mathrm{pw}}\left(z^{*} ; \sigma, \lambda_{\mathrm{w}}\right)$ can be approximated by $u_{\mathrm{pw}}\left(z^{*} ; \sigma, \lambda_{\mathrm{w}}\right)=-\varepsilon_{\mathrm{w}}$. $A_{2 \mathrm{D}}$ is the Helmholtz free energy of a two-dimensional fluid interacting via the pair potential $\phi(x, y)$. According to the standard Thermodynamic Perturbation Theory (TPT) of Zwanzig [34], we can express $A_{2 \mathrm{D}}$ as

$$
\frac{A_{2 \mathrm{D}}}{N_{\mathrm{ads}} k_{\mathrm{B}} T}=\ln \left(\rho_{\mathrm{ads}} \Lambda^{2}\right)-1+a_{\mathrm{HD}}+\beta a_{1}^{2 \mathrm{D}}+\beta^{2} a_{2}^{2 \mathrm{D}}+\ldots
$$

where $\rho_{\text {ads }}=\rho_{2 \mathrm{D}}$ corresponds to the number of molecules per unit area in the adsorbed phase, $a_{\mathrm{HD}}$ is the Helmholtz free energy of the reference system of a hard-disk fluid, and $a_{1}^{2 \mathrm{D}}$ and $a_{2}^{2 \mathrm{D}}$ are the first- and second-order perturbation terms.

In the present manuscript we restrict our discussion to the second-order perturbation expansion,

$$
\frac{A_{2 \mathrm{D}}}{N_{\mathrm{ads}} k_{\mathrm{B}} T}=\ln \left(\rho_{\mathrm{ads}} \Lambda^{2}\right)-1+a_{\mathrm{HD}}+\beta a_{1}^{2 \mathrm{D}}+\beta^{2} a_{2}^{2 \mathrm{D}} .
$$

The total Helmholtz free energy for the adsorbed fluid in Eq. (16) can be rewritten as

$$
\frac{A_{\mathrm{ads}}}{N_{\mathrm{ads}} k_{\mathrm{B}} T}=\frac{A_{2 \mathrm{D}}}{N_{\mathrm{ads}} k_{\mathrm{B}} T}-\ln \left(\frac{\lambda_{\mathrm{w}} \sigma}{\Lambda}\right)-\beta \varepsilon_{\mathrm{w}} .
$$

For the case of the bulk fluid, we consider an analogous perturbation expression given by

$$
\frac{A_{3 \mathrm{D}}}{N_{\mathrm{b}} k_{\mathrm{B}} T}=\ln \left(\rho_{\mathrm{b}} \Lambda^{3}\right)-1+a_{\mathrm{HS}}+\beta a_{1}^{3 \mathrm{D}}+\beta^{2} a_{2}^{3 \mathrm{D}}
$$

where $N_{\mathrm{b}}$ is the number of molecules in the bulk phase, $\rho_{\mathrm{b}}=\rho_{3 \mathrm{D}}$ is the corresponding density, $a_{\mathrm{HS}}$ is the 
Helmholtz free energy of the hard-sphere fluid and $a_{1}^{3 \mathrm{D}}$ and $a_{2}^{3 \mathrm{D}}$ are the first two perturbation terms.

The calculation of adsorption isotherms at temperature $T$ is performed by solving the equilibrium between the adsorbed and bulk phases, by equating the corresponding chemical potentials, i.e.,

$$
\mu_{\mathrm{ads}}=\mu_{\mathrm{b}}
$$

where

$$
\mu_{\mathrm{ads}}=\left(\frac{\partial A_{\mathrm{ads}}}{\partial N_{\mathrm{ads}}}\right)_{T, s}
$$

and

$$
\mu_{\mathrm{b}}=\left(\frac{\partial A_{\mathrm{b}}}{\partial N_{\mathrm{b}}}\right)_{T, V}
$$

in Eq. (23) $A_{\mathrm{b}} \equiv A_{3 \mathrm{D}}$ is the Helmholtz free energy of the $3 \mathrm{D}$ bulk fluid and the terms $N_{\mathrm{ads}}$ and $N_{\mathrm{b}}$ are the number of particles of the adsorbed and bulk phases, respectively $\left(N_{\text {ads }}+N_{\mathrm{b}}=N\right)$.

In previous communications $[19,22]$ we have tested with Monte Carlo computer simulations the quasi-2D approach described here when applied to the SW potential model, by considering 3D particles adsorbed in surfaces. It was found that the 2D-SAFT-VR approach gives very accurate adsorption isotherms when compared with $\mathrm{MC}$ values. This agreement is not due to the pair potential model used but to the actual decoupling approximation in the adsorbed particles pair potential and to the values of the adsorption wall-particle energy, and we can expect that the same result applies to the Mie potential.

\section{A. 2D SAFT-VR Mie}

In this section we present an equation of state for a $2 \mathrm{D}$ Mie fluid, based on the method followed in the SAFT-VR Mie approach [28]. The Helmholtz free energy for the 2D Mie fluid is given by

$$
\frac{A^{2 \mathrm{DMie}}}{N_{\mathrm{ads}} k_{\mathrm{B}} T}=\ln \left(\rho_{\mathrm{ads}} \Lambda^{2}\right)-1+a_{\mathrm{HD}}+\beta a_{1}^{2 \mathrm{DMie}}+\beta^{2} a_{2}^{2 \mathrm{DMie}}
$$

where the properties of the hard-disk reference fluid are obtained from a temperature-dependent effective diameter $d$, according to the Barker and Henderson perturbation theory [30],

$$
d=\int_{0}^{\sigma}(1-\exp (-\beta u(r))) d r .
$$

The first-order perturbation term is given by

$$
a_{1}^{2 \mathrm{DMie}}=\pi \rho_{2 \mathrm{D}} \int_{\sigma}^{\infty} u^{\mathrm{Mie}}(r) g_{\mathrm{HD}}(r) r \mathrm{~d} r
$$

where $g_{\mathrm{HD}}(r)$ is the hard-disk radial distribution function. The mean-attractive energy $a_{1}^{2 \mathrm{DMie}}$ can be obtained either by molecular simulation or by integration of $g_{\mathrm{HD}}$. In this work we have explored the analytical evaluation in the spirit of the SAFT-VR approach for 3D systems [28], using an approximated expression for $g_{\mathrm{HD}}$ given by Santos and Yuste [35], which expresses this property as a combination of $1 \mathrm{D}$ and $3 \mathrm{D}$ hard-body pair-correlation functions,

$$
\begin{aligned}
g_{\mathrm{HD}}(r ; \gamma) & =\alpha(\gamma) g_{1 \mathrm{D}}\left(r ; \omega_{1}(\gamma) \gamma_{1 D}\right) \\
& +(1-\alpha(\gamma)) g_{3 \mathrm{D}}\left(r ; \omega_{3}(\gamma) \gamma_{3 \mathrm{D}}\right)
\end{aligned}
$$

where three parameters are used to relate the properties of the systems with different dimensionality. The packing fractions are denoted by $\gamma_{1 \mathrm{D}}, \gamma$ and $\gamma_{3 \mathrm{D}}$ for 1,2 and 3 dimensions, respectively. The terms $\omega_{1}(\gamma)$ and $\omega_{3}(\gamma)$ are the scaling parameters for the packing fraction in the 1D and 3D space, respectively and the function $\alpha(\gamma)$ is a mixing parameter. These parameters can be determined numerically using the procedure outlined by Yuste and Santos [35]:

1. The contact value of the HD radial distribution function, $g_{\mathrm{HD}}(1, \gamma)$, corresponds to the hard-rod or hardsphere radial distribution function when $\alpha=1$ or $\alpha=0$, respectively. This enables us to obtain the parameters $\omega_{1}$ and $\omega_{3}$.

2. From the isothermal compressibility expression

$$
k_{\mathrm{B}} T\left(\frac{\partial \rho}{\partial P}\right)_{T}=1+2 \pi \rho \int_{0}^{\infty}\left[g_{\mathrm{HD}}(r, \gamma)-1\right] r \mathrm{~d} r
$$

and the heuristic expression for $g_{\mathrm{HD}}$ given in Eq. (27), it is possible to obtain $\alpha$ in terms of the corresponding expressions for the 2D and 3D terms, which can be determined numerically from molecular simulations or integral equations.

Once the parameters used in Eq. (27) are calculated, the scaling parameters are found to be smooth functions of $\gamma$, that can be represented by quadratic polynomials:

$$
\begin{aligned}
\omega_{1}(\gamma) & =1.5539-0.22742 \gamma-0.31723 \gamma^{2} \\
\omega_{3}(\gamma) & =0.62911+0.1804 \gamma-0.18307 \gamma^{2} \\
\alpha(\gamma) & =0.40462-0.21235 \gamma+0.1754 \gamma^{2}
\end{aligned}
$$

Using Eqs. (26) and (27), the first-order perturbation term can be expressed as,

$$
\begin{aligned}
a_{1}^{2 \mathrm{DMie}} & =\pi \rho_{2 \mathrm{D}} \int_{d}^{\infty} u^{\mathrm{Mie}}(r) g_{\mathrm{HD}}(r) r \mathrm{~d} r \\
& -\pi \rho_{2 \mathrm{D}} \int_{d}^{\sigma} u^{\mathrm{Mie}}(r) g_{\mathrm{HD}}(r) r \mathrm{~d} r \\
& =I_{1 \mathrm{~A}}+I_{1 \mathrm{~B}} .
\end{aligned}
$$

Following the SAFT-VR Mie method used to evaluate $a_{1}^{3 \mathrm{DMie}}[28]$, we can use an analogous procedure in the $2 \mathrm{D}$ case to obtain expressions for $I_{1 \mathrm{~A}}$ and $I_{1 \mathrm{~B}}$, as we explain in the following subsections. 


\section{B. Expression for $I_{1 \mathrm{~A}}$}

The first integral in Eq. (32) is

$$
I_{1 \mathrm{~A}}=\pi \rho_{2 \mathrm{D}} \int_{d}^{\infty} g_{\mathrm{HD}}(r) C \varepsilon\left[\left(\frac{\sigma}{r}\right)^{\lambda_{\mathrm{r}}}-\left(\frac{\sigma}{r}\right)^{\lambda_{\mathrm{a}}}\right] r \mathrm{~d} r .
$$

Defining the reduced distance $x$ between disk centers as

$$
x=\frac{r}{d}
$$

Eq. (33) transforms into

$$
I_{1 \mathrm{~A}}=\pi \rho_{2 \mathrm{D}} d^{2} C \varepsilon \int_{1}^{\infty} g_{\mathrm{HD}}(x d)\left[\left(\frac{x_{0}}{x}\right)^{\lambda_{\mathrm{r}}}-\left(\frac{x_{0}}{x}\right)^{\lambda_{\mathrm{a}}}\right] x \mathrm{~d} x
$$

where $x_{0}=\sigma / d$. The expression

$$
\begin{aligned}
a_{1}^{\mathrm{s}}(\gamma, \lambda) & =\pi \rho_{\mathrm{D}} \varepsilon d^{2} \int_{1}^{\infty}\left(-\frac{1}{x^{\lambda}}\right) g_{\mathrm{HD}}(x d) x \mathrm{~d} x \\
& =4 \gamma \varepsilon \int_{1}^{\infty}\left(-\frac{1}{x^{\lambda}}\right) g_{\mathrm{HD}}(x d) x \mathrm{~d} x
\end{aligned}
$$

represents the first-order term $a_{1}^{\mathrm{s}}(\gamma ; \lambda)$ of the Helmholtz free energy of a system of hard-core Sutherland particles of diameter $d$ characterized by the range parameter $\lambda$. From Eqs. (26) and (36) we obtain

$$
\begin{aligned}
a_{1}^{\mathrm{s}}(\gamma ; \lambda) & =-4 \gamma \varepsilon \alpha \int_{1}^{\infty} g_{1 \mathrm{D}}\left(\omega_{1} \gamma\right) x^{1-\lambda} \mathrm{d} x \\
& -4 \gamma \varepsilon(1-\alpha) \int_{1}^{\infty} g_{3 \mathrm{D}}\left(\omega_{3} \gamma\right) x^{1-\lambda} \mathrm{d} x
\end{aligned}
$$

The 1D and 3D packing fractions have here been expressed simply as $\gamma$ because it is easy to distinguish between them by looking at the corresponding multiplying scaling parameter.

Eq. (37) can be further separated into two integrals.

$$
a_{1}^{\mathrm{s}}(\gamma ; \lambda)=I_{2 \mathrm{~A}}+I_{2 \mathrm{~B}}
$$

where

$$
I_{2 \mathrm{~A}}=-4 \gamma \varepsilon \alpha \int_{1}^{\infty} g_{1 \mathrm{D}}\left(\omega_{1} \gamma\right) x^{1-\lambda} \mathrm{d} x
$$

and

$$
I_{2 \mathrm{~B}}=-4 \gamma \varepsilon(1-\alpha) \int_{1}^{\infty} g_{3 \mathrm{D}}\left(\omega_{3} \gamma\right) x^{1-\lambda} \mathrm{d} x .
$$

An exact expression for the hard-rod radial distribution function $g_{1 \mathrm{D}}$ is given by the Salsburg-Zwanzig-Kirkwood solution [36],

$g_{1 \mathrm{D}}(x ; \rho \sigma)=\frac{1}{\rho \sigma} \sum_{k=1}^{\infty} \frac{W^{k} e^{W k}}{(k-1) !}(x-k)^{k-1} e^{-W x} H(x-k)$

where $H(x-k)$ is the Heaviside Function,

$$
H(x-k)= \begin{cases}1 & \text { if } x \geq k \\ 0 & \text { if } x<k\end{cases}
$$

and $W=\rho \sigma /(1-\rho \sigma)$. By substituting $g_{1 \mathrm{D}}$ in $I_{2 \mathrm{~A}}$ and exploiting the properties of the Heaviside function, we obtain

$$
\begin{aligned}
& \int_{1}^{\infty} g_{1 \mathrm{D}}\left(x ; \omega_{1} \gamma\right) x^{1-\lambda} \mathrm{d} x= \\
& \quad \frac{1}{\omega_{1} \gamma} \sum_{k=1}^{\infty} \frac{W^{k} e^{W k}}{(k-1)} \int_{k}^{\infty}(x-k)^{k-1} x^{1-\lambda} e^{-W x} \mathrm{~d} x
\end{aligned}
$$

A final expression for $I_{2 \mathrm{~A}}$ is derived

1) using the binomial expansion of order $k-1$

$$
(x-k)^{k-1}=\sum_{j=0}^{\infty}\left(\begin{array}{c}
j, \\
k-1
\end{array}\right) x^{k-1-j}(-k)^{j}
$$

where the term $\sum_{j=0}^{\infty}\left(\begin{array}{c}j, \\ k-1\end{array}\right)$ is a series from $j$ to $k-1$, and

2) introducing the incomplete $\Gamma$ function,

$$
\Gamma(\alpha, s)=\int_{s}^{\infty} u^{\alpha-1} e-u \mathrm{~d} u .
$$

With this, one obtains

$$
\begin{aligned}
I_{2 \mathrm{~A}} & =-\frac{4 \gamma \varepsilon \alpha}{\omega_{1} \gamma} \sum_{k=1}^{\infty} \frac{W^{k} e^{W k}}{(k-1) !} \\
& \times \sum_{j=0}^{\infty} \frac{(j, k-1)(-k)^{j}}{W^{k-\lambda-j+1}} \Gamma(k-\lambda-j+1, W k) .
\end{aligned}
$$

For the three-dimensional case [28], we have the SAFTVR Mie analytical expression for $a_{1}{ }^{\mathrm{s}-3 \mathrm{D}}(\eta ; \lambda)$

$$
\int_{1}^{\infty} g_{3 \mathrm{D}}(x) x^{2-\lambda} \mathrm{d} x=-\frac{a_{1}^{\mathrm{s}-3 \mathrm{D}}(\eta ; \lambda)}{12 \eta \varepsilon} .
$$

By comparing this result with $I_{2 \mathrm{~B}}$, we then have

$$
I_{2 \mathrm{~B}}=\frac{(1-\alpha)}{3 \omega_{3}} a_{1}{ }^{\mathrm{s}-3 \mathrm{D}}\left(\omega_{3} \gamma ; \lambda+1\right)
$$

where the term $a_{1}^{\mathrm{s}-3 \mathrm{D}}(\eta ; \lambda)$ is the first-order term of the Helmholtz free energy of a three-dimensional system of hard-core Sutherland particles. This term was derived previously $[18,28]$. Accordingly, the $2 \mathrm{D}$ first-order term of the hard-core Sutherland system is

$$
\begin{aligned}
a_{1}^{\mathrm{s}}(\gamma ; \lambda) & =-\frac{4 \gamma \varepsilon \alpha}{\omega_{1} \gamma} \sum_{k=1}^{\infty} \frac{W^{k} e^{W k}}{(k-1) !} \\
& \times \sum_{j=0}^{\infty} \frac{(j, k-1)(-k)^{j}}{W^{k-\lambda-j+1}} \Gamma(k-\lambda-j+1, W k) \\
& +\frac{(1-\alpha)}{3 \omega_{3}} a_{1}^{\mathrm{s}-3 \mathrm{D}}\left(\omega_{3} \gamma ; \lambda+1\right)
\end{aligned}
$$

and integral $I_{1 \mathrm{~A}}$ is

$$
I_{1 \mathrm{~A}}=C\left(x_{0}^{\lambda_{\mathrm{a}}} a_{1}^{\mathrm{s}}\left(\gamma ; \lambda_{\mathrm{a}}\right)-x_{0}^{\lambda_{\mathrm{r}}} a_{1}^{\mathrm{s}}\left(\gamma ; \lambda_{\mathrm{r}}\right)\right) .
$$




\section{Expression for $I_{1 \mathrm{~B}}$}

In order to calculate the integral $I_{1 \mathrm{~B}}$, the radial distribution function $g_{\mathrm{HD}}$ is approximated with a linear relation obtained from a first-order Taylor expansion of the contact value of $g_{\mathrm{HD}}(r)$ and its first derivative with respect to the distance $r$

$$
g_{\mathrm{HD}}(x d) \approx g_{\mathrm{HD}}(d)+(x-1)\left(\frac{\mathrm{d} g_{\mathrm{HD}}(x d)}{\mathrm{d} x}\right)_{x=1} .
$$

Therefore, we have

$$
\begin{aligned}
I_{1 \mathrm{~B}} & \approx-\pi \rho_{2 \mathrm{D}} d^{2} g_{\mathrm{HD}}(d) \int_{1}^{x_{0}} u^{\mathrm{Mie}}(x d) x \mathrm{~d} x \\
& -\pi \rho_{2 \mathrm{D}} d^{2}\left(\frac{\mathrm{d} g_{\mathrm{HD}}(x d)}{\mathrm{d} x}\right)_{1} \int_{1}^{x_{0}} u^{\mathrm{Mie}}(x d) x(x-1) \mathrm{d} x .
\end{aligned}
$$

In order to simplify this expression we write

$$
I_{\lambda}(\lambda)=\int_{1}^{x_{0}} \frac{x}{x^{\lambda}} \mathrm{d} x=-\frac{\left(x_{0}\right)^{2-\lambda}-1}{\lambda-2}
$$

and

$$
\begin{aligned}
J_{\lambda}(\lambda) & =\int_{1}^{x_{0}} \frac{x^{2}-x}{x^{\lambda}} \mathrm{d} x \\
& =-\frac{\left(x_{0}\right)^{3-\lambda}(\lambda-2)-\left(x_{0}\right)^{2-\lambda}(\lambda-3)-1}{(\lambda-3)(\lambda-2)} .
\end{aligned}
$$

The analytical expression of $I_{1 \mathrm{~B}}$ is then given by

$$
I_{1 \mathrm{~B}}=C\left(x_{0}^{\lambda_{\mathrm{a}}} B\left(\gamma ; \lambda_{\mathrm{a}}\right)-x_{0}^{\lambda_{\mathrm{r}}} B\left(\gamma ; \lambda_{\mathrm{r}}\right)\right)
$$

where

$$
B(\gamma ; \lambda)=4 \gamma \varepsilon\left(\frac{1-\frac{7 \gamma}{16}}{(1-\gamma)^{2}} I_{\lambda}(\lambda)-\Phi(\gamma) J_{\lambda}(\lambda)\right) ;
$$

the contact value of the HD radial distribution function is obtained from the Henderson EOS [38]

$$
g_{\mathrm{HD}}=\frac{1-\frac{7 \gamma}{16}}{(1-\gamma)^{2}}
$$

and

$$
\begin{aligned}
\Phi(\gamma) & =\left(\frac{\mathrm{d} g_{\mathrm{HD}}(x d)}{\mathrm{d} x}\right)_{1} \\
& =-\alpha \frac{\omega_{1} \gamma}{\left(1-\omega_{1} \gamma\right)}-4.5(1-\alpha) \omega_{3} \gamma{\frac{\left(1+\omega_{3} \gamma\right)^{3}}{\left(1-\omega_{3} \gamma\right)}}^{.}
\end{aligned}
$$

Finally, the analytical expression for the first-order perturbation term for the $2 \mathrm{D}$ Mie fluid is

$$
\begin{aligned}
a_{1}^{2 \text { DMie }} & =C\left[x_{0}^{\lambda_{\mathrm{a}}}\left(a_{1}^{s}\left(\gamma ; \lambda_{\mathrm{a}}\right)+B\left(\gamma ; \lambda_{\mathrm{a}}\right)\right)\right] \\
& -C\left[x_{0}^{\lambda_{\mathrm{r}}}\left(a_{1}^{s}\left(\gamma ; \lambda_{\mathrm{r}}\right)+B\left(\gamma ; \lambda_{\mathrm{r}}\right)\right)\right] .
\end{aligned}
$$

As can be seen from this equation, the treatment of the analytical expression of $a_{1}^{2 \text { DMie }}$ seems to be particularly difficult since it involves an infinite series over an incomplete gamma function, see Eq. (47). However, the expression can be approximated to the first term, $k=1$, that uses the hard-rod radial distribution function from $r=0$ to $r=2 \sigma$, and a correction term is introduced assuming $g_{\mathrm{HD}}(r) \approx 1$ for $r>2 \sigma$,

$$
\begin{aligned}
a_{1, \mathrm{corr}}^{2 \mathrm{DMie}} & =\pi \rho_{2 \mathrm{D}} \int_{2 \sigma}^{\infty} u^{\mathrm{Mie}}(r) r \mathrm{~d} r \\
& =4 C \gamma \varepsilon\left[\left(\frac{2^{\left(2-\lambda_{\mathrm{a}}\right)}}{2-\lambda_{\mathrm{a}}}\right)-\left(\frac{2^{\left(2-\lambda_{\mathrm{r}}\right)}}{2-\lambda_{\mathrm{r}}}\right)\right] .
\end{aligned}
$$

By introducing this correction, the resulting analytical expression of $a_{1}^{2 D M i e}$ is given by

$$
\begin{aligned}
a_{1}^{2 \text { DMie }} & =C\left[x_{0}^{\lambda_{\mathrm{a}}}\left(a_{1}^{s}\left(\gamma ; \lambda_{\mathrm{a}}\right)+B\left(\gamma ; \lambda_{\mathrm{a}}\right)\right)\right] \\
& -C\left[x_{0}^{\lambda_{\mathrm{r}}}\left(a_{1}^{s}\left(\gamma ; \lambda_{\mathrm{r}}\right)+B\left(\gamma ; \lambda_{\mathrm{r}}\right)\right)\right] \\
& +4 C \gamma \varepsilon\left[\left(\frac{2^{\left(2-\lambda_{\mathrm{a}}\right)}}{2-\lambda_{\mathrm{a}}}\right)-\left(\frac{2^{\left(2-\lambda_{\mathrm{r}}\right)}}{2-\lambda_{\mathrm{r}}}\right)\right] .
\end{aligned}
$$

In the case of the second-order perturbation term, an expression based on the 3D SAFT-VR-Mie theory and the macroscopic compressibility approximation [37] has been derived for the $2 \mathrm{D}$ case,

$$
a_{2}^{2 \mathrm{DMie}}=\frac{1}{2} K_{\mathrm{HD}}\left(1+2 \psi \gamma^{2}\right) a_{1}^{*}
$$

where $K_{\mathrm{HD}}$ is the hard-disk isothermal compressibility,

$$
K_{\mathrm{HD}}=\frac{(1-\gamma)^{3}}{1+\gamma+0.375 \gamma^{2}-0.125 \gamma^{3}},
$$

obtained from the Henderson EOS [38]; $\psi$ is a 2D correction term of the macroscopic compressibility approximation [39],

$$
\psi=\psi_{0}\left(1-7.63944 x_{0}^{2} \gamma+17.83253 x_{0}^{4} \gamma^{2}\right)
$$

where $\psi_{0}=1 / \gamma_{0}^{2}$ and $\gamma_{0}=0.69015$ is the HD packing fraction for the liquid phase in the liquid-solid transition, and $a_{1}^{*}$ is the first-order perturbation term for the square of the Mie potential,

$$
a_{1}^{*}=\pi \rho_{2 \mathrm{D}} \int_{\sigma}^{\infty}\left[u^{\mathrm{Mie}}(r)\right]^{2} g_{\mathrm{HD}}(r) r d r .
$$

The last quantity is easily evaluated with the expression that we have developed for $a_{1}^{2 \mathrm{DMie}}$ considering the square of the pair potential (see Appendix C). The Barker and Henderson compressibility approximation was originally derived for 3D fluids [37], and assumes that the fluctuations in energy are correlated to the fluctuation of the number of interacting particles inside the attractive well of the pair potential, an effect that is approximated by the isothermal compressibility of the entire fluid. The correction introduced by Zhang [39] modifies this assumption by considering the high-density limit of HS packing of particles in the liquid phase. The 2D version presented here gives the standard compresibility approximation (2D MCA) by taking $\psi=0$ in Eq. (60). 


\section{Comparison with MC simulation results for $a_{1}^{2 \mathrm{DMie}}$ and $a_{2}^{2 \mathrm{DMie}}$}

Standard Metropolis Monte Carlo (MC) simulations at constant number of particles, area and temperature ( $N s T$ ensemble) were performed in order to numerically compute the first- and second-order perturbation terms of a set of 2D Mie fluids with different repulsive and attractive exponents $\left(\lambda_{\mathrm{r}}\right.$ and $\left.\lambda_{\mathrm{a}}\right)$. Systems at several values of reduced density $\left(\rho_{2 \mathrm{D}}^{*}=\rho_{2 \mathrm{D}} \sigma^{2}\right)$ were simulated at reduced temperatures $T^{*}=k_{\mathrm{B}} T / \varepsilon=1$ and $T^{*}=2$. Simulations were carried out using 864 particles, contained within a $2 \mathrm{D}$ square box, with $2.5 \times 10^{5}$ cycles required for thermalisation and $5.0 \times 10^{5}$ cycles for statistics (the number of cycles is defined as the number of movements per particle). Runs were performed with a $40-50 \%$ acceptance ratio.

In Figs. 2-5 results are presented for $a_{1}^{2 D M i e}$ and $a_{2}^{2 \mathrm{DMie}}$ obtained from theory and MC simulations for Mie fluids with selected values of the repulsive exponent $\lambda_{\mathrm{r}}$, fixing the attractive exponent to the London value of $\lambda_{\mathrm{a}}=6$. The corresponding numerical values are summarized in Tables IV-IX of Appendix A, where we include as a comparison parameter the percent deviation of the theoretical data with respect to the MC values, $\% \mathrm{D}=\frac{a_{n}^{\text {Theory }}-a_{n}^{\mathrm{MC}}}{a_{n}^{\mathrm{MC}}} \times 100$.

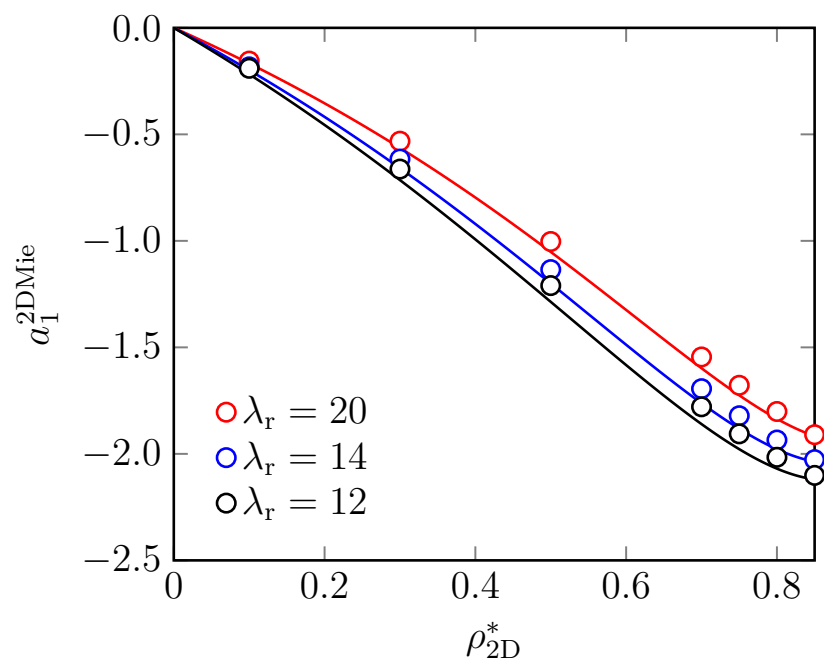

Figure 2: The density $\rho_{2 \mathrm{D}}^{*}$ dependence of the first-order Barker and Henderson perturbation term $a_{1}^{2 D M i e}$ at a reduced temperature $T^{*}=1$, for Mie fluids with exponents $\lambda_{\mathrm{a}}=6$ and $\lambda_{\mathrm{r}}$ and as indicated. Continuous curves are obtained from Eq. (59) whereas the circles represent the exact results obtained by MC computer simulation.

Good agreement between $a_{1}^{2 D M i e}$ values obtained using the theoretical expression and the corresponding $\mathrm{MC}$ simulation values is found for densities $\rho_{2 \mathrm{D}}^{*}<0.85$. Theoretical and $\mathrm{MC}$ values diverge from each other at higher densities, corresponding to packing fractions higher than

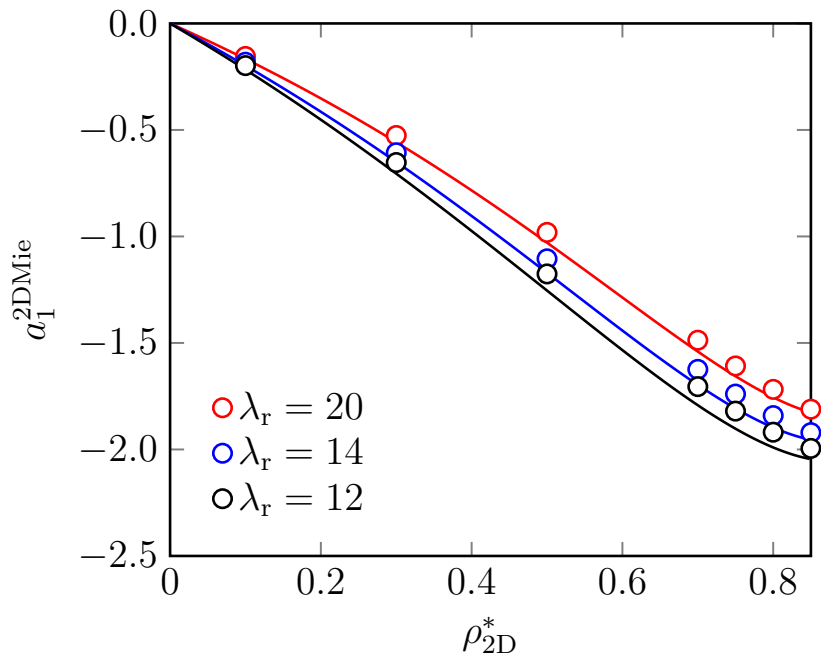

Figure 3: The density $\rho_{2 \mathrm{D}}^{*}$ dependence of the first-order Barker and Henderson perturbation term $a_{1}^{2 \mathrm{DMie}}$ at a reduced temperature $T^{*}=2$, for Mie fluids with exponents $\lambda_{\mathrm{a}}=6$ and $\lambda_{\mathrm{r}}$ and as indicated. Continuous curves are obtained from Eq. (59) whereas the circles represent the exact results obtained by $\mathrm{MC}$ computer simulation.

0.67. From MC simulation studies [40] it is known that the hard-disk fluid undergoes a fluid-solid transition when $\gamma \approx 0.69$ in the fluid branch. In other words, the divergence found for the first-order perturbation term may be an indication of the HD liquid-solid phase-transition.

The results obtained for $a_{2}^{2 \mathrm{DMie}}$ can be seen from Figs. 4 and 5, and are in good agreement with the exact MC values. The trends obtained with density and temperature are correct, and in better agreement than the MCA approximation (see also Tables IV-IX in Appendix A).

The approach followed in this article modifies the previous version based on the SW potential. The use of the Mie potential widens the scope of applications with this potential model, since for bulk phases the SAFT-VR-Mie has been used to reproduce and characterise a larger variety of thermodynamic properties of substances than the $\mathrm{SW}$ version. The use of the bulk parameters is required to describe adsorption isotherms, as explained in the following section. From the theoretical point of view, the $2 \mathrm{D}$ version for the Mie system uses a better representation of the second-order perturbation term; in the case of the SW theory, this term is given by the local compressibility approximation, which has a lower accuracy than the corrected MCA version introduced here.

\section{MODELLING ADSORPTION ISOTHERMS OF REAL FLUIDS: $\mathrm{CO}_{2}$ AND $\mathrm{CH}_{4}$}

The prediction of adsorption isotherms using the 3D/2D SAFT-VR Mie approach is based on the solution of the thermodynamic equilibrium condition. Thus, 


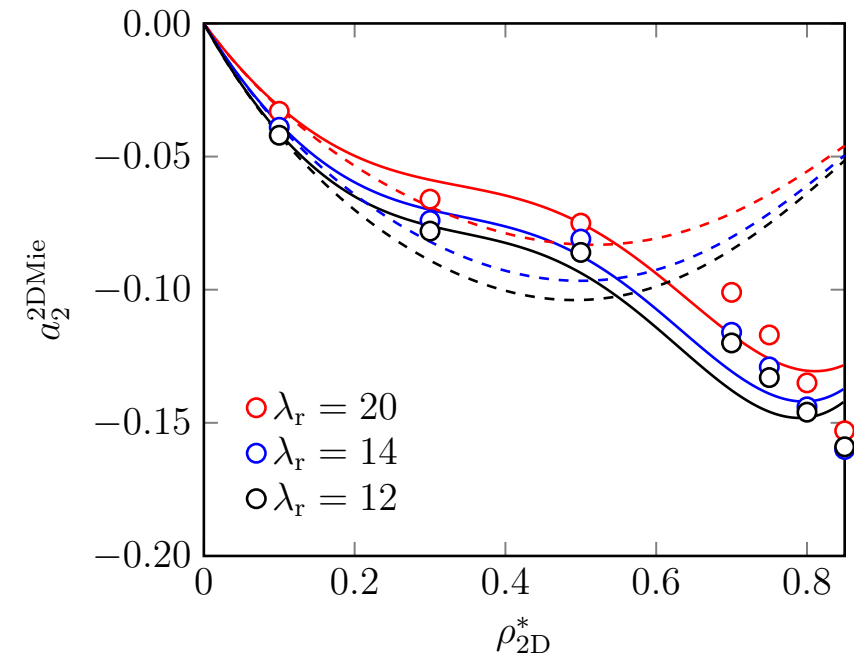

Figure 4: Values of $a_{2}^{2 D M i e}$ as function of the 2D reduced density $\rho_{2 D}^{*}$ obtained using the 2D SAFT-VR Mie, the 2D Macroscopic Compressibility Approximation (2D MCA), and MC simulations for Mie fluids with exponents $\lambda_{\mathrm{a}}=6$ and several values of $\lambda_{\mathrm{r}}$, for a reduced temperature $T^{*}=1.0$. Continuous curves corresponds to Eqs. (60)-(63) and dashed lines are the 2D MCA values, based on the original expression by Barker and Henderson for 3D fluids, which is obtained by taking $\psi=0$ in the aforementioned equations. MC computer simulation results are represented by circles.

coexisting densities are obtained by demanding equality in the chemical potentials of the adsorbed and bulk phases, for a given temperature, $T$, and bulk pressure, $P$, as expressed in Eq. (21).

The chemical potential for a pure fluid is calculated from the standard thermodynamic relationship

$$
\beta \mu_{\mathrm{b}}=a_{3 \mathrm{D}}+\eta\left(\frac{\partial a_{3 \mathrm{D}}}{\partial \eta}\right)_{V, T}
$$

and

$$
\beta \mu_{\mathrm{ads}}=a_{\mathrm{ads}}+\gamma\left(\frac{\partial a_{\mathrm{ads}}}{\partial \gamma}\right)_{s, T}
$$

where $\eta$ and $\gamma$ are the 3D and 2D molar packing fractions, respectively. From Eq. (64) and the 3D/2D SAFT-VR Mie approach, the equations to be considered to model adsorption isotherms are:

$$
\beta \mu_{\mathrm{ads}}=\beta \mu_{2 \mathrm{D}}+\beta \mu_{\mathrm{w}}
$$

where

$$
\beta \mu_{2 \mathrm{D}}=\ln (\gamma)+a_{\mathrm{HD}}+\beta a_{1}^{2 \mathrm{DMie}}+\beta^{2} a_{2}^{2 \mathrm{DMie}}+\frac{\beta P_{2 \mathrm{D}}}{\rho_{2 \mathrm{D}}}
$$

and

$$
\beta \mu_{\mathrm{w}}=\ln (2 / 3)-\ln \left(\lambda_{\mathrm{w}}\right)-\beta \varepsilon_{\mathrm{w}}
$$

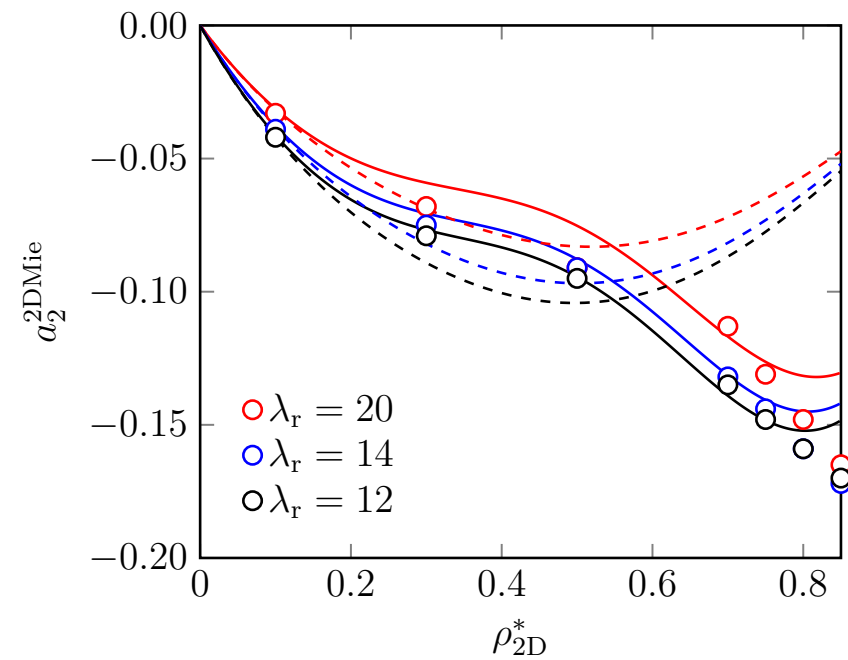

Figure 5: Values of $a_{2}^{2 \mathrm{DMie}}$ as function of the 2D reduced density $\rho_{2 D}^{*}$ obtained using the 2D SAFT-VR Mie, the 2D Macroscopic Compressibility Approximation (2D MCA), and MC simulations for Mie fluids with exponents $\lambda_{\mathrm{a}}=6$ and several values of $\lambda_{\mathrm{r}}$, for a reduced temperature $T^{*}=2.0$. Continuous curves correspond to Eqs. (60)-(63) and dashed lines are the 2D MCA values, based on the original expression by Barker and Henderson for 3D fluids, which is obtained by taking $\psi=0$ in the aforementioned equations. MC computer simulation results are represented by circles.

$$
\begin{aligned}
\beta \mu_{\mathrm{b}} & =\ln (\eta)+a_{\mathrm{HS}}+\beta a_{1}^{3 \mathrm{DM} i \mathrm{e}} \\
& +\beta^{2} a_{2}^{3 \mathrm{DMie}}+\beta^{3} a_{3}^{3 \mathrm{DMie}}+\frac{\beta P_{3 \mathrm{D}}}{\rho_{3 \mathrm{D}}} .
\end{aligned}
$$

The terms $P_{3 \mathrm{D}}$ and $P_{2 \mathrm{D}}$ correspond to the three- and two-dimensional pressures respectively. The Helmholtz free energies of the hard-disk and hard-sphere reference systems are given by the Henderson [38], and CarnahanStarling [41] equations of state, respectively:

$$
\begin{gathered}
\frac{A_{\mathrm{HD}}}{N_{\mathrm{ads}} k_{\mathrm{B}} T}=a_{\mathrm{HD}}=\frac{9 \gamma}{8(1-\gamma)}-\frac{7}{8} \ln (1-\gamma) \\
\frac{A_{\mathrm{HS}}}{N_{\mathrm{b}} k_{\mathrm{B}} T}=a_{\mathrm{HS}}=\frac{4 \eta-3 \eta^{2}}{(1-\eta)^{2}} .
\end{gathered}
$$

The molecular parameters of the 3D/2D SAFT-VR Mie approach for calculating adsorption isotherms of model fluids are:

1. The particle-particle interaction in the bulk phase: $\sigma_{\mathrm{b}}, \varepsilon_{\mathrm{b}}^{\mathrm{Mie}}, \lambda_{\mathrm{r}}, \lambda_{\mathrm{a}}$.

2. The particle-particle interaction in the adsorbed phase:

$\sigma_{\mathrm{ads}}, \varepsilon_{\mathrm{ads}}^{\mathrm{Mie}}, \lambda_{\mathrm{r}}^{\mathrm{ads}}, \lambda_{\mathrm{a}}^{\mathrm{ads}}$.

3. The particle-wall interaction:

$\varepsilon_{\mathrm{w}}$ and $\lambda_{\mathrm{w}}$. 
In order to test the accuracy of the theory, the cases of the adsorption of carbon dioxide $\left(\mathrm{CO}_{2}\right)$ and methane $\left(\mathrm{CH}_{4}\right)$ onto dry coal were considered. Molecular parameters were defined under the following assumptions:

1. Bulk parameters $\left(\sigma_{\mathrm{b}}, \varepsilon_{\mathrm{b}}^{\mathrm{Mie}}, \lambda_{\mathrm{r}}, \lambda_{\mathrm{a}}\right)$. Parameters reported in Table I were adopted to model the bulk phases of $\mathrm{CO}_{2}$ [27] and $\mathrm{CH}_{4}$ [28]. The values of these parameters are independent of the adsorption process.

2. The molecules in the adsorbed and bulk phases have the same segment diameter, i.e., $\sigma_{\mathrm{ads}}=\sigma_{\mathrm{b}}$.

3. The adsorbed-phase particle-particle Mie-potential parameters are the same as in the bulk phase, $\lambda_{\mathrm{r}}^{\mathrm{ads}}=\lambda_{\mathrm{r}}$ and $\lambda_{\mathrm{a}}^{\mathrm{ads}}=\lambda_{\mathrm{a}}$, i.e., the shape of the pair interaction is the same for particles in both phases.

4. The ratio of critical temperatures of the adsorbed and bulk phases, $R_{\mathrm{c}}=T_{\mathrm{c}}^{\mathrm{ads}} / T_{\mathrm{c}}^{\mathrm{b}}$ is approximated to 0.4 . This value corresponds to the ratio obtained from experimental data of adsorption of fluids [15], and to the 2D/3D ratios obtained from the Ising model [42] and computer simulation data of Lennard-Jones fluids [43, 44]. This criterion implies, using the 2D and 3D SAFT-VR Mie EoS, that

$$
\varepsilon_{\mathrm{ads}} / \varepsilon_{\mathrm{b}}=0.4 \frac{T_{c(\mathrm{~b})}^{*}}{T_{c(\mathrm{ads})}^{*}}=0.8097 .
$$

This value is also in agreement with the prediction given by Sinanonoglu and Pitzer [45].

5. The particle-wall interaction parameters i.e., $\varepsilon_{\mathrm{w}}$ and $\lambda_{\mathrm{w}}$ are determined by numerical fitting to provide the best resproduction of experimental adsorption data, subject to the constraint that $0.1305 \leq \lambda_{\mathrm{w}} \leq 0.8165$, in order to guarantee monolayer adsorption [16].

6. The specific surface area $\left(a_{s}\right)$ of adsorbent is also obtained by regression. In the present case, the value for dry coal was constrained to lie within the range of values provided in the experimental study of adsorption of $\mathrm{CO}_{2}$ and $\mathrm{CH}_{4}$ on dry coal by Ottiger et al. [46].

Table I: Values of 3D SAFT-VR Mie parameters for pair interactions in the bulk phase

\begin{tabular}{lcccc}
\hline \hline Substance & $\left(\varepsilon_{\mathrm{b}}^{\text {Mie }} / k_{\mathrm{B}}\right) / \mathrm{K}$ & $\sigma / \AA$ & $\lambda_{\mathrm{r}}$ & $\lambda_{\mathrm{a}}$ \\
\hline $\mathrm{CO}_{2}[27]$ & 353.5500 & 3.7410 & 23.0000 & 6.6600 \\
$\mathrm{CH}_{4}[28]$ & 153.3600 & 3.7412 & 12.6500 & 6.0000 \\
\hline
\end{tabular}

Table II: The SAFT-VR Mie scheme for the modelling of adsorption isotherms of real single-component fluids

\begin{tabular}{ll}
\hline \hline $\begin{array}{l}\text { Interaction } \\
\text { Particle-particle, } \\
\text { bulk phase }\end{array}$ & $\sigma, \lambda_{\mathrm{r}}, \lambda_{\mathrm{a}}, \varepsilon_{\mathrm{b}}$ \\
$\begin{array}{l}\text { Particle-particle, } \\
\text { adsorbed phase }\end{array}$ & $\sigma, \lambda_{\mathrm{r}}, \lambda_{\mathrm{a}}, \varepsilon_{\mathrm{ads}}$ \\
Particle-wall & $\lambda_{\mathrm{w}}, \varepsilon_{\mathrm{w}}$ \\
\hline Parameter & Origin \\
\hline$\sigma, \lambda_{\mathrm{r}}, \lambda_{\mathrm{a}}, \varepsilon_{\mathrm{b}}$ & $\begin{array}{l}\text { Ref. [27] } \\
\text { from } R_{c}, \text { via Eq. (72) }\end{array}$ \\
$\varepsilon_{\mathrm{ads}}$ & $\begin{array}{l}\text { obtained by fitting to } \\
\lambda_{\mathrm{w}}, \varepsilon_{\mathrm{w}}\end{array}$ \\
\hline
\end{tabular}

Table III: Optimised parameters for modelling of adsorption of $\mathrm{CO}_{2}$ and $\mathrm{CH}_{4}$ onto dry coal

\begin{tabular}{lcccc}
\hline \hline System & $\left(\varepsilon_{\mathrm{ads}}^{\text {Mie }} / k_{\mathrm{B}}\right) / \mathrm{K}$ & $\lambda_{\mathrm{w}}$ & $\varepsilon_{\mathrm{w}} / \varepsilon_{\mathrm{b}}^{\text {Mie }}$ & $a_{s} /\left(\mathrm{m}^{2} \mathrm{~g}_{\mathrm{s}}^{-1}\right)$ \\
\hline $\mathrm{CO}_{2}(318.16 \mathrm{~K})$ & 286.26 & 0.61 & 4.02 & 322.95 \\
$\mathrm{CO}_{2}(333.16 \mathrm{~K})$ & 286.26 & 0.62 & 3.98 & 310.45 \\
$\mathrm{CH}_{4}(318.16 \mathrm{~K})$ & 124.17 & 0.13 & 13.35 & 191.52 \\
\hline
\end{tabular}

In Table II the procedure to determine the molecular parameters for modelling of adsorption of real fluids is summarised. The optimised parameter values used in the modelling of adsorption isotherms of $\mathrm{CO}_{2}$ and $\mathrm{CH}_{4}$ onto dry coal are reported in Table III.

In Fig. 6, experimental and theoretical results are presented for the adsorption isotherms of $\mathrm{CO}_{2}$ onto dry coal at two different temperatures, namely $318.16 \mathrm{~K}$ and $333.16 \mathrm{~K}$. In particular, we report the number of adsorbed moles per unit mass of adsorbent $\left(n / m_{\mathrm{s}}\right)$ as a function of bulk density. The experimental values are compiled from the work of Ottiger et al. [46] and the theoretical adsorption isotherms are calculated by solving the thermodynamic equilibrium condition between bulk and adsorbed phases. The transformation of the coexisting densities to the commonly used experimental units is discussed in Appendix B. As observed, the theory allows for an excellent reproduction of the experimental values in the whole range of bulk fluid densities. Additionally, the variation of the $\left(n / m_{\mathrm{s}}\right)\left(\rho_{\text {bulk }}\right)$ curve with temperature is also well captured. It is worth noting that the increase to a maximum value and subsequent quasi-linear decrease on the adsorbed amount with increasing density is a characteristic feature of the adsorption of $\mathrm{CO}_{2}$ on dry coal $[46,47]$.

As in the case of the 2D-SAFT-VR for SW particles, the extension to the Mie potential can be used to accurately describe a wide range of substances. The case of adsorption of $\mathrm{CH}_{4}$ on dry coal at $318.16 \mathrm{~K}$ is reported in Fig. 7 , where agreement between experimental measurements [46] and theory is also attained. These observations illustrate the capabilities and predictive power of the theory as well as the quality of the molec- 


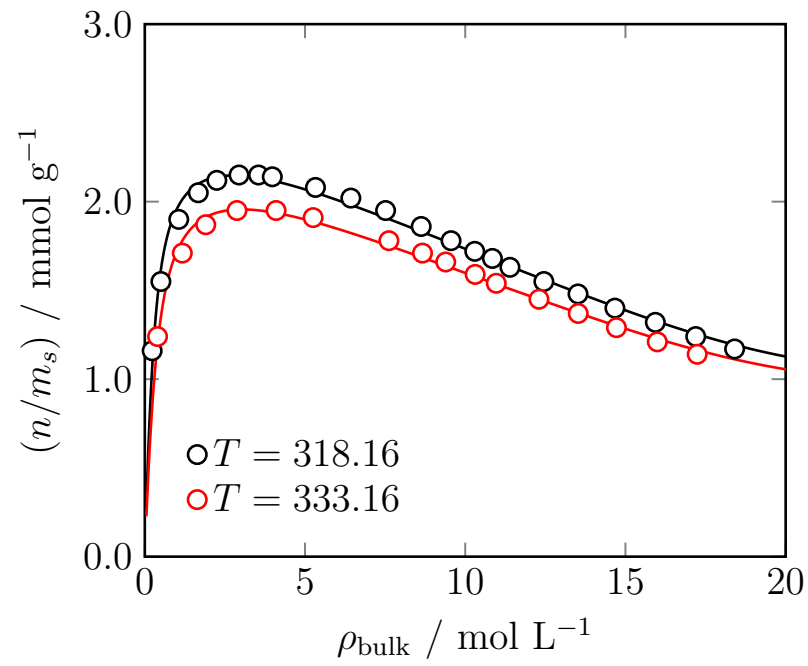

Figure 6: Adsorbed amount $\left(n / m_{\mathrm{s}}\right)$ as a function of bulk density $\left(\rho_{\text {bulk }}\right)$ for $\mathrm{CO}_{2}$ onto dry coal at $318.16 \mathrm{~K}$ and $333.16 \mathrm{~K}$. Comparison between the theoretical descriptions (continuous curves) and the experimental values (circles) reported in Ref. [46].

ular parameters used for describing both fluid-fluid and fluid-solid interactions. In further communications we will present applications to associating systems and their mixtures.

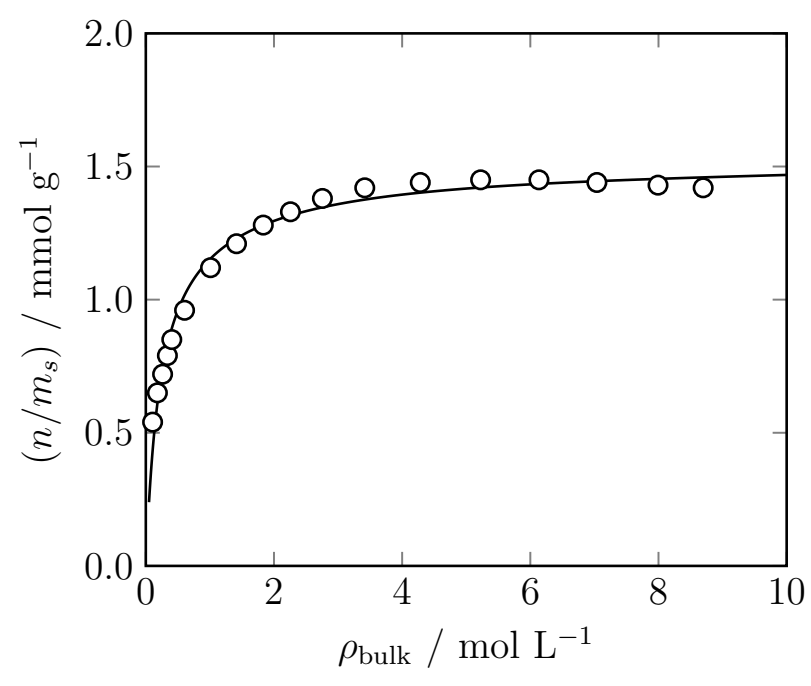

Figure 7: Adsorbed amount $\left(n / m_{\mathrm{s}}\right)$ as a function of bulk density $\left(\rho_{\text {bulk }}\right)$ for $\mathrm{CH}_{4}$ onto dry coal at $318.16 \mathrm{~K}$. Comparison between the theoretical description (continuous curve) and the experimental values (circles) reported in Ref. [46]

\section{CONCLUSIONS}

In this work we have presented the 2D SAFT-VR modelling of adsorption isotherms for particles interacting via Mie pair-potentials, based on the extension to the case of two-dimensional fluids of the SAFT-VR Mie approach [28]. By considering the examples of $\mathrm{CO}_{2}$ and $\mathrm{CH}_{4}$ adsorbed onto dry coal, we have shown that excellent results can be obtained from the application of the theory, even considering that the Mie pair interaction in the adsorbed and bulk phases have the same shape, given by the parameters $\lambda_{\mathrm{a}}$ and $\lambda_{\mathrm{r}}$. This result is in contrast with the 2D SAFT-VR SW version, that requires non-conformal pair interactions in the adsorbed and bulk phases [20-22]. In this way, the use of the Mie potential simplifies the application of the SAFT approach to model adsorption. The application of this theory to the case of associating and multicomponent systems will be the subject of future publications.

\section{ACKNOWLEDGMENTS}

This work was carried out in part as an activity of the Qatar Carbonates and Carbon Storage Research Centre (QCCSRC). We gratefully acknowledge funding from QCCSRC provided jointly by Qatar Petroleum, Shell, and the Qatar Science and Technology Park. We thank the Universidad de Guanajuato (México) for financial support via DAIP-UG projects CIIC 1143/2016 and CIIC 292/2018. GCV is also thankful to the University of Guanajuato for a students mobility grant for a research visit to Imperial College London.
[1] A. W. Adamson, A. P. Gast, Physical Chemistry of surfaces. John Wiley and Sons, 6th Edition, New York
(1997).

[2] United States Environmental Protection Agency, Inven- 
tory of U.S. Greenhouse Gas Emisions and Sinks, 19902016, https://www.epa.gov/ghgemissions/inventory-usgreenhouse-gas-emissions-and-sinks, (2018).

[3] I. Langmuir, J. Am. Chem. Soc. 40, 1361 (1918).

[4] S. Brunauer, P. Emmet and E. Teller, J. Am. Chem. Soc. 60, 309 (1938).

[5] G. Cornelissen, D. W. Rutherford, H. P. H. Arp, P. Dorsch, C. N. Kelly and C. E. Rostad, Environ. Sci. \& Technol. 47, 7704 (2013).

[6] H. Furukawa, F. Gándara, Y. B. Zhang, J. Jiang, W. L. Queen, M. R. Hudson and O. M. Yaghi, J. Am. Chem. Soc. 136, 4369 (2014).

[7] C. S. Dutcher, X. Ge, A. S. Wexler, S. L. Clegg, J. Phys. Chem. C 115, 16474 (2011).

[8] C. S. Dutcher, X. Ge, A. S. Wexler, S. L. Clegg, J. Phys. Chem. C 116, 1850 (2012).

[9] E. A. Muller, L. F. Rull, L. F. Vega and K. E. Gubbins, J. Phys. Chem. 100, 1189 (1996).

[10] Q. Yang, and C. Zhong, J. Phys. Chem. B 110, 17776 (2006).

[11] L. Sarkisov and P. A. Monson, Langmuir 17, 7600 (2001).

[12] J. H. deBoer, The dynamical character of adsorption, Oxford: Clarendon Press (1953).

[13] T. L. Hill, J. Chem. Phys. 17, 520 (1949).

[14] W. D. Machin and S. Ross, Proc. R. Soc. Lond. A 265 , 455 (1962).

[15] J. G. Dash, Films on Solid Surfaces. Academic Press, New York (1975).

[16] F. del Río and A. Gil-Villegas, J. Phys. Chem. 95,787 (1991).

[17] C. Zhou, F. Hall, K. A. M. Gasem,R. L. Robinson Jr., Ind. Eng. Chem. Res. 33, 1280 (1994).

[18] A. Gil-Villegas, A. Galindo, P. J. Whitehead, S. Mills, G. Jackson and A. Burgess, J. Chem. Phys. 106, 4168 (1997).

[19] A. Martínez, M. Castro, C. McCabe and A. Gil-Villegas, J. Chem. Phys. 126, 074707 (2007).

[20] G. Jiménez-Serratos, S. Santillán, C. Avendano, M. Castro and A. Gil-Villegas, Oil and Gas Sci. Tech. 63, 329 (2008).

[21] M. Castro, A. Martínez and A. Gil-Villegas, Adsorpt. Sci. Technol. 29, 59 (2011).

[22] V. M. Trejos, M. Becerra, S. Figueroa-Gerstenmaier and A. Gil-Villegas, Mol. Phys. 112, 2330 (2014).

[23] A. Martínez, V. M. Trejos and A. Gil-Villegas, Fluid Phase Equil. 449, 207 (2017).

[24] J. Kern and M. Johannsen, J. Supercritical Fluids 133, 70 (2018).

[25] M. Castro, J. L. Mendoza de la Cruz, E. BuenrostroGonzález, S. López-Ramírez and A. Gil-Villegas, Fluid Phase Equil. 87, 113 (2009).

[26] G. Mie, Ann. Phys. (Berlin) 316, 657 (1903).

[27] C. Avendaño, T. Lafitte, A. Galindo, C. S. Adjiman, G. Jackson, E. Müller, J. Phys. Chem. B 115, 11154 (2011).

[28] T. Lafitte, A. Apostolakou, C. Avendaño, A. Galindo, C. S. Adjiman, E. Müller, G. Jackson, J. Chem. Phys. 139, 154504 (2013).

[29] L. F. Franco, I. G. Economou and M. Castier, Langmuir 33, 11291 (2017).

[30] J. A. Barker, D. Henderson, J. Chem. Phys. 47, 2856 (1967).

[31] V. M. Trejos, A. Santos and F. Gámez, J. Chem. Phys 148, 194505 (2018)

[32] G. Campos-Villalobos, Developing a classical 2D SAFT-
$V R$ Mie approach to the prediction of adsorption isotherms of $\mathrm{CO}_{2}$ and other fluids, Bachelor Thesis in Chemical Engineering, University of Guanajuato, México (2016).

[33] R. C. Tolman, J. Chem. Phys. 16, 758 (1948).

[34] R. W. Zwanzig, J. Chem. Phys. 22, 1420 (1954).

[35] S. B. Yuste and A. Santos, J. Chem. Phys. 101, 2355 (1994).

[36] Z. W. Salsburg, R. W. Zwanzig and J. G. Kirkwood, J. Chem. Phys. 21, 1098 (1953).

[37] J. A. Barker, D. Henderson, J. Chem. Phys. 47, 2856 (1967).

[38] D. Henderson, Mol. Phys. 30, 971 (1975).

[39] B.-J. Zhang, Fluid Phase Eq. 154,1 (1999).

[40] B. J. Alder and T. E. Wainwright, J. Chem. Phys. 1208, (1957).

[41] N. Carnahan, K. E. Starling, J. Chem. Phys. 51, 635 (1969).

[42] C. Domb, Adv. Phys. 9, 245 (1960).

[43] D. Henderson, Mol. Phys. 34, 301 (1977).

[44] N. B. Wilding, Phys. Rev. E 52, 602 (1995)

[45] O. Sinanoglu, K. S. Pitzer, J. Chem. Phys. 32, 1279 (1960).

[46] S. Ottiger, R. Pini, G. Storti, M. Mazzotti, R. Bencini, F. Quattrocchi, G. Sardu, G. Deriu, Environ. Prog. 25, 355 (2006).

[47] S. Ottiger, R. Pini, G. Storti, M. Mazzotti, Adsorption 14, 539 (2008). 


\section{Appendix A: Tables}

In this and the following Appendices we provide information complementary to the results discussed in this work.

In Tables IV-IX we report information about the comparison between the theoretical values of perturbation terms and their corresponding Monte Carlo computersimulation values.

Table IV: Values of $a_{1}^{2 \mathrm{DMie}}$ and $a_{2}^{2 \mathrm{DMie}}$ for a Mie fluid characterized by the exponents $\lambda_{\mathrm{r}}=12$ and $\lambda_{\mathrm{a}}=6$ at a reduced temperature $T^{*}=1$. Comparison between theory and MC computer simulations.

\begin{tabular}{rrrrrrr}
\hline \hline$\rho_{2 \mathrm{D}}^{*}$ & $a_{1}^{2 \mathrm{DMie}}$ & $\mathrm{MC}$ & $\% D$ & $a_{2}^{2 \mathrm{DMie}}$ & $\mathrm{MC}$ & $\% D$ \\
\hline 0.10 & -0.218 & -0.190 & 14.605 & -0.041 & -0.042 & 2.071 \\
0.30 & -0.715 & -0.663 & 7.802 & -0.076 & -0.078 & 2.555 \\
0.50 & -1.286 & -1.210 & 6.308 & -0.094 & -0.086 & 8.970 \\
0.70 & -1.860 & -1.779 & 4.565 & -0.138 & -0.120 & 14.818 \\
0.75 & -1.978 & -1.906 & 3.770 & -0.146 & -0.133 & 9.654 \\
0.80 & -2.069 & -2.016 & 2.644 & -0.148 & -0.146 & 1.368 \\
0.85 & -2.120 & -2.101 & 0.890 & -0.142 & -0.159 & 10.784 \\
\hline \hline
\end{tabular}

Table V: Values of $a_{1}^{2 \mathrm{DMie}}$ and $a_{2}^{2 \mathrm{DMie}}$ for a Mie fluid characterized by the exponents $\lambda_{\mathrm{r}}=14$ and $\lambda_{\mathrm{a}}=6$, at a reduced temperature $T^{*}=1$. Comparison between theory and MC computer simulations.

\begin{tabular}{crrrrrr}
\hline \hline$\rho_{2 \mathrm{D}}^{*}$ & $a_{1}^{2 \mathrm{DMie}}$ & $\mathrm{MC}$ & $\% D$ & $a_{2}^{2 \mathrm{DMie}}$ & $\mathrm{MC}$ & $\% D$ \\
\hline 0.10 & -0.199 & -0.183 & 8.643 & -0.038 & -0.039 & 3.470 \\
0.30 & -0.659 & -0.616 & 6.948 & -0.070 & -0.074 & 5.406 \\
0.50 & -1.200 & -1.135 & 5.742 & -0.087 & -0.081 & 7.796 \\
0.70 & -1.762 & -1.694 & 4.034 & -0.131 & -0.116 & 12.659 \\
0.75 & -1.882 & -1.821 & 3.351 & -0.139 & -0.129 & 7.801 \\
0.80 & -1.978 & -1.935 & 2.232 & -0.142 & -0.144 & 1.412 \\
0.85 & -2.036 & -2.027 & 0.460 & -0.137 & -0.160 & 14.349 \\
\hline \hline
\end{tabular}

Table VI: Values of $a_{1}^{2 \mathrm{DMie}}$ and $a_{2}^{2 \mathrm{DMie}}$ for a Mie fluid characterized by the exponents $\lambda_{\mathrm{r}}=20$ and $\lambda_{\mathrm{a}}=6$, at a reduced temperature $T^{*}=1$. Comparison between Theory and MC computer simulations.

\begin{tabular}{rrrrrrr}
\hline \hline$\rho_{2 \mathrm{D}}^{*}$ & $a_{1}^{2 \mathrm{DMie}}$ & $\mathrm{MC}$ & $\% D$ & $a_{2}^{2 \mathrm{DMie}}$ & $\mathrm{MC}$ & $\% D$ \\
\hline 0.10 & -0.167 & -0.156 & 7.144 & -0.031 & -0.033 & 5.411 \\
0.30 & -0.564 & -0.532 & 5.957 & -0.059 & -0.066 & 11.007 \\
0.50 & -1.052 & -1.003 & 4.883 & -0.075 & -0.075 & 0.072 \\
0.70 & -1.598 & -1.545 & 3.427 & -0.117 & -0.101 & 15.710 \\
0.75 & -1.725 & -1.678 & 2.803 & -0.126 & -0.117 & 7.629 \\
0.80 & -1.836 & -1.801 & 1.916 & -0.130 & -0.135 & 3.377 \\
0.85 & -1.916 & -1.910 & 0.329 & -0.128 & -0.153 & 16.219 \\
\hline \hline
\end{tabular}

Table VII: Values of $a_{1}^{2 \mathrm{DMie}}$ and $a_{2}^{2 \mathrm{DMie}}$ for a Mie fluid characterized by the exponents $\lambda_{\mathrm{r}}=12$ and $\lambda_{\mathrm{a}}=6$, at a reduced temperature $T^{*}=2$. Comparison between Theory and MC computer simulations.

\begin{tabular}{rrrrrrr}
\hline \hline$\rho_{2 \mathrm{D}}^{*}$ & $a_{1}^{2 \mathrm{DMie}}$ & $\mathrm{MC}$ & $\% D$ & $a_{2}^{2 \mathrm{DMie}}$ & $\mathrm{MC}$ & $\% D$ \\
\hline 0.10 & -0.217 & -0.198 & 9.550 & -0.041 & -0.042 & 1.793 \\
0.30 & -0.705 & -0.653 & 7.992 & -0.077 & -0.079 & 2.796 \\
0.50 & -1.254 & -1.176 & 6.643 & -0.094 & -0.095 & 0.586 \\
0.70 & -1.792 & -1.705 & 5.125 & -0.139 & -0.135 & 3.017 \\
0.75 & -1.903 & -1.820 & 4.539 & -0.148 & -0.148 & 0.194 \\
0.80 & -1.991 & -1.919 & 3.730 & -0.152 & -0.159 & 4.274 \\
0.85 & -2.045 & -1.995 & 2.530 & -0.148 & -0.170 & 12.746 \\
\hline \hline
\end{tabular}

Table VIII: Values of $a_{1}^{2 \text { DMie }}$ and $a_{2}^{2 \text { DMie }}$ for a Mie Fluid characterized by the exponents $\lambda_{\mathrm{r}}=14$ and $\lambda_{\mathrm{a}}=6$, at a reduced temperature $T^{*}=2$. Comparison between Theory and MC computer simulations.

\begin{tabular}{rrrrrrr}
\hline \hline$\rho_{2 \mathrm{D}}^{*}$ & $a_{1}^{2 \mathrm{DMie}}$ & $\mathrm{MC}$ & $\% D$ & $a_{2}^{2 \mathrm{DMie}}$ & $\mathrm{MC}$ & $\% D$ \\
\hline 0.10 & -0.198 & -0.182 & 8.846 & -0.038 & -0.039 & 3.235 \\
0.30 & -0.650 & -0.607 & 7.157 & -0.071 & -0.075 & 5.846 \\
0.50 & -1.171 & -1.105 & 5.969 & -0.088 & -0.091 & 3.501 \\
0.70 & -1.697 & -1.624 & 4.491 & -0.131 & -0.132 & 0.440 \\
0.75 & -1.808 & -1.740 & 3.880 & -0.141 & -0.144 & 2.309 \\
0.80 & -1.897 & -1.841 & 3.051 & -0.145 & -0.159 & 8.830 \\
0.85 & -1.955 & -1.921 & 1.754 & -0.142 & -0.172 & 17.525 \\
\hline \hline
\end{tabular}

Table IX: Values of $a_{1}^{2 \mathrm{DMie}}$ and $a_{2}^{2 \mathrm{DMie}}$ for a Mie Fluid characterized by the exponents $\lambda_{\mathrm{r}}=20$ and $\lambda_{\mathrm{a}}=6$, at a reduced temperature $T^{*}=2$. Comparison between Theory and MC computer simulations.

\begin{tabular}{rrrrrrr}
\hline \hline$\rho_{2 \mathrm{D}}^{*}$ & $a_{1}^{2 \mathrm{DMie}}$ & $\mathrm{MC}$ & $\% D$ & $a_{2}^{2 \text { DMie }}$ & $\mathrm{MC}$ & $\% D$ \\
\hline 0.100 & -0.167 & -0.155 & 7.520 & -0.031 & -0.033 & 5.249 \\
0.300 & -0.558 & -0.526 & 6.020 & -0.059 & -0.068 & 13.097 \\
0.500 & -1.029 & -0.981 & 4.921 & -0.075 & -0.091 & 17.315 \\
0.700 & -1.540 & -1.487 & 3.592 & -0.117 & -0.113 & 3.413 \\
0.750 & -1.656 & -1.608 & 2.991 & -0.126 & -0.131 & 3.555 \\
0.800 & -1.755 & -1.718 & 2.163 & -0.132 & -0.148 & 11.074 \\
0.850 & -1.826 & -1.811 & 0.848 & -0.130 & -0.165 & 20.963 \\
\hline \hline
\end{tabular}




\section{Appendix B: Quantitative expression of adsorption to compare with experimental data values}

In order to quantify the amount of adsorbed particles the Gibbs' concept of surface excess is used [33]; this consists of an imaginary surface placed parallel to the solid wall of the adsorbent. The surface excess amount, $n$, corresponds to the number of moles of the adsorbed fluid. The amount $n$ is an extensive quantity, which depends on the extent of the interface and can be related to the surface excess concentration, $\Gamma$, by the following relationship

$$
\frac{\Gamma}{N_{\mathrm{A}}}=\frac{n}{s_{s}}
$$

where $N_{\mathrm{A}}$ is the Avogadro's number and $s_{\mathrm{s}}$ is the surface area associated with the mass of solid adsorbent $m_{\mathrm{s}}$. The specific surface area $a_{\mathrm{s}}$ is therefore

$$
a_{\mathrm{s}}=\frac{s_{\mathrm{s}}}{m_{\mathrm{s}}} .
$$

In many cases, adsorption data values are reported using the quantity $\left(n / m_{\mathrm{s}}\right)$, termed the specific surface excess amount, and defined as the ratio of the surface excess amount over the solid mass (adsorbent mass). Therefore, a compact expression for the specific surface excess amount or the amount adsorbed is given by

$$
\frac{n}{m_{\mathrm{s}}}=\frac{\Gamma a_{\mathrm{s}}}{N_{\mathrm{A}}} .
$$

In this equation, the only undefined term is the surface excess concentration, $\Gamma$. This term is defined as

$$
\Gamma=\int_{0}^{\infty}\left[\rho(z)-\rho_{\mathrm{b}}\right] \mathrm{d} z
$$

where $\rho(z)$ is the density of particles in the adsorbed phase and $\rho_{\mathrm{b}}$ is the bulk density of particles, i.e., $\rho(z \rightarrow \infty)=\rho_{\mathrm{b}}$. The surface excess concentration is commonly called the Gibbs surface excess concentration, denoted as $\Gamma_{\text {Gibbs }}$. In the case of a solid wall interacting with the adsorbed particles via a SW potential, we have that

$$
\Gamma_{\text {Gibbs }}=\int_{0}^{\lambda_{\mathrm{w}} \sigma} \rho(z) \mathrm{d} z-\rho_{\mathrm{b}} \lambda_{\mathrm{w}} \sigma
$$

where $\lambda_{\mathrm{w}} \sigma$ is the length scale of the adsorbed phase. The integral in this equation is the absolute adsorption concentration, $\Gamma_{\text {abs }}$, defined as the total concentration of adsorbed molecules, given by

$$
\Gamma_{\mathrm{abs}}=\rho_{\mathrm{ads}}=\int_{0}^{\lambda_{\mathrm{w}} \sigma} \rho(z) \mathrm{d} z
$$

where $\rho_{\text {ads }}=\rho_{2 \mathrm{D}}$ is the density of molecules of adsorbed fluid. This relation can also be written as

$$
\Gamma_{\text {Gibbs }} \sigma^{2}=\Gamma_{\text {abs }} \sigma^{2}-6 \eta \lambda_{\mathrm{w}} / \pi
$$

where $\Gamma_{\text {abs }} \sigma^{2}=4 \gamma / \pi$. The Gibbs surface excess concentration and the absolute adsorption concentration values can be calculated when the equilibrium conditions are satisfied. Therefore, the connection with the experimental data values is accomplished by the calculation of the adsorbed amount, given by

$$
n / m_{\mathrm{s}}=\frac{\left(\Gamma_{\mathrm{Gibbs}} \sigma^{2}\right) a_{\mathrm{s}}}{N_{\mathrm{A}} \sigma^{2}} .
$$




\section{Appendix C: Evaluation of $a_{1}^{*}$}

From simple algebraic manipulation, it can be demonstrated that the mean-attractive energy for the square of the Mie pair potential, $a_{1}^{*}$, required in the evaluation of $a_{2}^{2 \mathrm{DMie}}(\mathrm{Eq} .(61))$ is given by:

$$
\begin{aligned}
a_{1}^{*} & =C^{2}\left[x_{0}^{2 \lambda_{\mathrm{a}}}\left(a_{1}^{s}\left(\gamma ; 2 \lambda_{\mathrm{a}}\right)+B\left(\gamma ; 2 \lambda_{\mathrm{a}}\right)\right)\right] \\
& +C^{2}\left[x_{0}^{2 \lambda_{\mathrm{r}}}\left(a_{1}^{s}\left(\gamma ; 2 \lambda_{\mathrm{r}}\right)+B\left(\gamma ; 2 \lambda_{\mathrm{r}}\right)\right)\right] \\
& -2 C^{2}\left[x_{0}^{\lambda_{\mathrm{r}}+\lambda_{\mathrm{a}}}\left(a_{1}^{s}\left(\gamma ; \lambda_{\mathrm{r}}+\lambda_{\mathrm{a}}\right)+B\left(\gamma ; \lambda_{\mathrm{r}}+\lambda_{\mathrm{a}}\right)\right)\right] \\
& +4 C^{2} \gamma \varepsilon^{2}\left[2\left(\frac{2^{\left(2-\left(\lambda_{\mathrm{r}}+\lambda_{\mathrm{a}}\right)\right)}}{2-\left(\lambda_{\mathrm{r}}+\lambda_{\mathrm{a}}\right)}\right)-\left(\frac{2^{\left(2-2 \lambda_{\mathrm{r}}\right)}}{2-2 \lambda_{\mathrm{r}}}\right)-\left(\frac{2^{\left(2-2 \lambda_{\mathrm{a}}\right)}}{2-2 \lambda_{\mathrm{a}}}\right)\right] .
\end{aligned}
$$

